\title{
Bases neuroanatómicas del síndrome de Wallenberg
}

\section{Neuroanatomical basis of Wallenberg syndrome}

\author{
Oscar O. Gasca-González ${ }^{1,2 *}$, Julio C. Pérez-Cruz ${ }^{2,3}$, Matias Baldoncini4, Mario A. Macías-Duvignau ${ }^{3}$ y \\ Luis Delgado-Reyes 2,5 \\ ${ }^{1}$ Clínica de Medicina Familiar Santa María, Instituto de Seguridad y Servicios Sociales para los Trabajadores del Estado, Ciudad de México, México; \\ ${ }^{2}$ Facultad de Medicina, Universidad Autónoma de México (UNAM), Ciudad de México, México; ${ }^{3}$ Academia de Anatomía, Escuela Superior de \\ Medicina, Instituto Politécnico Nacional, Ciudad de México, México; ${ }^{4}$ Laboratorio de Neuroanatomía Microquirúrgica, LaNeMic-ll Cátedra de \\ Anatomía, Facultad de Medicina, Universidad de Buenos Aires, Buenos Aires, Argentina; ${ }^{5}$ Departamento de Anatomía, Departamento de Cirugía, \\ Hospital Juárez de México, Facultad de Medicina, UNAM, Ciudad de México, México
}

\section{Resumen}

El síndrome de Wallenberg, o síndrome bulbar lateral, es la manifestación clínica del infarto en el territorio de irrigación de la arteria cerebelosa posteroinferior. Su presentación incluye vértigo, nistagmo, diplopía, síndrome de Horner, rubicundez y anhidrosis facial homolateral, disfonía, disfagia, disartria, pérdida homolateral del reflejo nauseoso, ataxia homolateral, disgeusia homolateral, dolor y parestesia faciales homolaterales, pérdida o disminución homolateral del reflejo corneal, hipoalgesia y termoanestesia de tronco y extremidades contralaterales, hipoalgesia y termoanestesia facial homolateral. El conocimiento neuroanatómico es imprescindible para su comprensión, estudio y diagnóstico, ya que sus manifestaciones neurológicas clásicas son fácilmente explicables y entendibles si se conocen la función y la localización de las estructuras anatómicas afectadas, así como la irrigación cerebral posterior.

Palabras Clave: Síndrome de Wallenberg. Isquemia. Médula oblongada. Bulbo raquídeo.

\begin{abstract}
Wallenberg syndrome, or lateral medullar syndrome, is the clinical presentation of the infarct in the territory of posterior inferior cerebellar artery. Its signs and symptoms include vertigo, nystagmus, diplopia, ipsilateral Horner syndrome, facial ruddiness and dry skin, dysphonia, dysphagia, dysarthria, ipsilateral loss of gag reflex, ipsilateral ataxia, ipsilateral impaired taste, ipsilateral facial pain and paresthesia, decreased ipsilateral blink reflex, contralateral hypoalgesia and thermoanaesthesia in the trunk and limbs; and ipsilateral facial hypoalgesia and thermoanaesthesia. Neuroanatomical knowledge is essential to its comprehension, study and diagnosis, because the classic neurological manifestations are easy to explain and understand if function and localization of affected anatomical structures are known as if the posterior cerebral circulation is.
\end{abstract}

Key Words: Wallenberg. Syndrome. Ischemia. Medulla oblongata.

\footnotetext{
Correspondencia:

*Oscar O. Gasca-González

José Antonio Álzate, 168

Col. Santa María la Ribera, Del. Cuauhtémoc

Fecha de recepción: 11-10-2018

C.P. 06400, Ciudad de México, México

E-mail: oogg1708@gmail.com

Cir Cir. 2020;88(3):376-382

Contents available at PubMed www.cirugiaycirujanos.com 0009-7411/@ 2019 Academia Mexicana de Cirugía. Publicado por Permanyer. Este es un artículo open access bajo la licencia CC BY-NC-ND (http://creativecommons.org/licenses/by-nc-nd/4.0/).
} 


\section{Introducción}

Descrito por primera vez en 1895 por el neurólogo polaco Adolf Wallenberg ${ }^{1,2}$, el síndrome de Wallenberg, también conocido como síndrome bulbar lateral, síndrome de la arteria cerebelosa posteroinferior (PICA, posterior inferior cerebellar artery), infarto medular lateral 0 infarto medular dorsolateral ${ }^{2-4}$, está causado por el infarto de la porción lateral y posterior al núcleo olivar inferior del bulbo raquídeo (médula oblongada) justo en el territorio irrigado por la PICA².

Aproximadamente el $83 \%$ de los eventos vasculares cerebrales en los Estados Unidos de América son de etiología isquémica; de estos, solo el $20 \%$ ocurren en el territorio vertebrobasilar ${ }^{2}$.

Las causas más comunes y descritas del síndrome de Wallenberg son, en orden de frecuencia, la oclusión aterotrombótica de la arteria vertebral, de la PICA o de las arterias medulares, cardioembolia y disección vertebral ${ }^{2,5}$. Sin embargo, existen otras causas menos comunes, pero bien descritas, del síndrome de Wallenberg, como lo son el uso de drogas emergentes conocidas como legal highs 0 "subidones legales» ${ }^{6}$, el consumo de cocaína ${ }^{7}$, la sarcoidosis ${ }^{8}$, la granulomatosis con poliangeítis (granulomatosis de Wegener) ${ }^{3}$, la arteritis de células gigantes ${ }^{9}$, la picadura de escorpión ${ }^{10}$ e incluso el aneurisma de PICA sin rotura ${ }^{11}$.

\section{Anatomía del bulbo raquídeo}

El bulbo raquídeo suele estudiarse en dos porciones, una abierta y otra cerrada, ambas separadas por el óbex, quedando cefálica a este la porción abierta (Fig. 1) que contiene diversas estructuras, tanto de sustancia blanca como de sustancia gris (Fig. 2).

\section{Núcleos}

Dentro de los núcleos que contiene el bulbo raquídeo en su porción abierta se encuentran los siguientes:

- Núcleo ambiguo: contiene las células encargadas de inervar los músculos del paladar blando, la faringe, la laringe y el esófago superior a través de los nervios craneales glosofaríngeo, vago y accesorio. Asimismo, contiene neuronas parasimpáticas que controlan la frecuencia cardiaca a través del nervio vago ${ }^{12,13}$.

- Núcleo del hipogloso: formado por las neuronas motoras que van a constituir el nervio hipogloso, da movimiento a la lengua. Las fibras neuronales provenientes de este núcleo salen por el surco preolivar, entre la pirámide y la oliva, para formar el nervio craneal XII.

- Núcleo dorsal del nervio vago: es el núcleo parasimpático más grande del tallo cerebral y está compuesto por neuronas motoras, cuyas fibras preganglionares formarán parte del nervio vago al salir por el surco retroolivar justo por detrás de la oliva. Su función incluye la regulación motora glandular y del músculo liso en las vísceras del tórax y el abdomen.

- Núcleo espinal del trigémino: las neuronas localizadas en este núcleo reciben aferencias homolaterales de los nervios trigémino, facial, glosofaríngeo y vago, a través del tracto espinal del trigémino. Sus fibras ascienden por el tracto trigémino-talámico ventral, mismo que se decusa en su trayecto hacia el tálamo contralateral. Su función es la transmisión de sensaciones generales (tacto, dolor y temperatura) de la cabeza ${ }^{12,13}$.

- Núcleos olivares: incluyen los núcleos olivar inferior, olivar accesorio medial y olivar accesorio dorsal, y forman parte de los llamados núcleos precerebelares. Estos núcleos reciben información del tracto espinoolivar proveniente del asta posterior contralateral de todos los segmentos medulares; asimismo, recibe información del núcleo rojo (a través del tracto tegmental dorsal) y de la corteza cerebral homolaterales. Sus fibras continúan hacia el cerebelo a través del pedúnculo cerebeloso inferior. Este complejo nuclear ayuda en la coordinación de patrones de movimiento aprendidos ${ }^{12,13}$.

- Núcleo solitario: recibe información a través del tracto solitario, proveniente de los ganglios inferior del hipogloso, vago y ganglio geniculado del nervio facial. La porción caudal de este núcleo participa en los reflejos viscerales relacionados con los nervios glosofaríngeo y vago (reflejos nauseoso y vagales), mientras que la porción cefálica recibe información del gusto proveniente de los nervios facial y glosofaríngeo.

- Núcleos vestibulares inferior y medial: contienen neuronas que reciben aferencias a través del nervio vestibulococlear. Algunas de sus fibras entran al cerebelo a través del pedúnculo cerebeloso inferior, otras descienden a través del tracto vestibuloespinal y otras más (provenientes en su mayoría del núcleo vestibular medial) forman parte del fascículo longitudinal medial. 


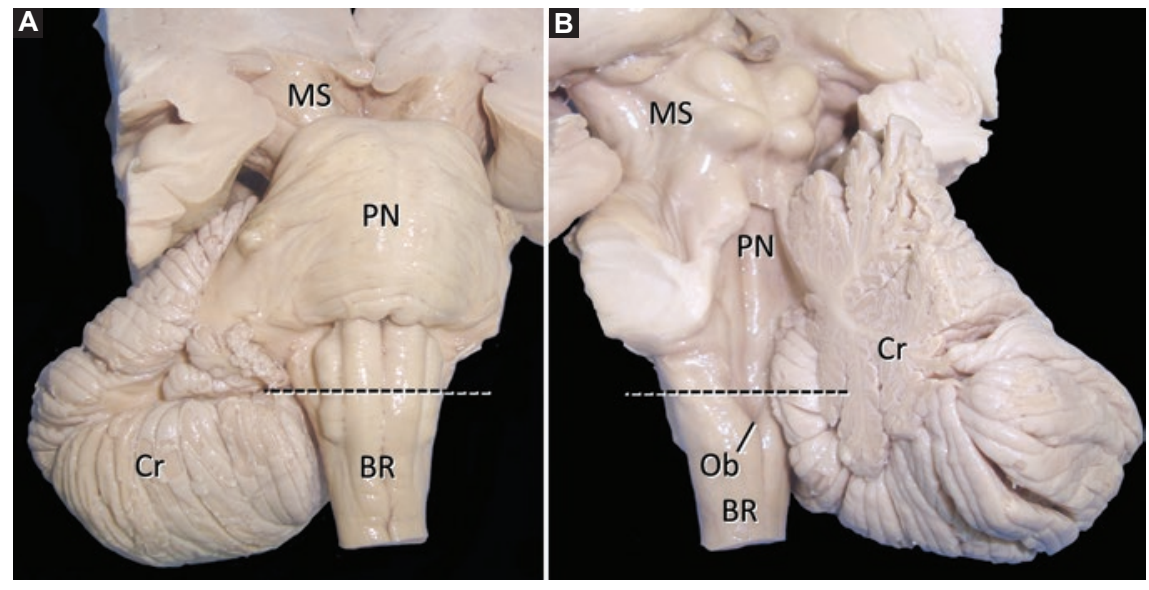

Figura 1. Anatomía externa del bulbo raquídeo. A: vista anterior del tallo cerebral. B: vista posterolateral izquierda del tallo cerebral. La línea discontinua muestra el nivel de corte de la figura 2. El hemisferio cerebeloso izquierdo fue cortado. BR: bulbo raquídeo; Cr: cerebelo; MS: mesencéfalo; Ob: óbex; PN: puente.

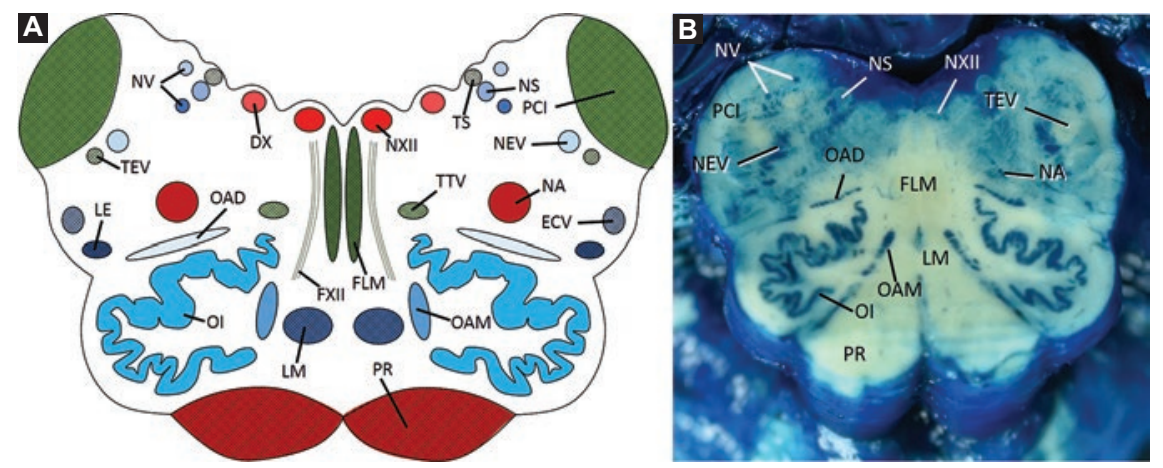

Figura 2. Anatomía interna del bulbo raquídeo (corte transversal, v. fig. 1). A: esquema anatómico. B: bulbo raquídeo humano con tinción de Mulligan. Núcleos motores (tonos rojos): núcleo dorsal del vago (DX), núcleo ambiguo (NA) y núcleo del hipogloso (NXII). Núcleos sensitivos (tonos azules): núcleo espinal del trigémino (NEV), núcleo solitario (NS), núcleos vestibulares inferior y medial (NV); núcleo olivar accesorio dorsal (OAD), núcleo olivar accesorio medial (OAM) y núcleo olivar inferior (OI). Tractos ascendentes (tonos azules con rayas): tracto espinocerebeloso ventral (ECV), lemnisco espinal (LE) y lemnisco medio (LM). Tractos descendentes (tonos rojos con rayas): tracto piramidal (PR). Otros tractos y fibras (tonos verdes con rayas): fascículo longitudinal medial (FLM), pedúnculo cerebeloso inferior (PCI), tracto espinal del trigémino (TEV), tracto solitario (TS), tracto trigeminotalámico ventral (TTV) y fibras del nervio hipogloso (FXII).

\section{Sustancia blanca}

En la porción abierta del bulbo raquídeo atraviesan los siguientes axones:

- Lemnisco medio: formado por las fibras ascendentes de los núcleos grácil y cuneiforme posterior a su decusación. Estas fibras terminan en la porción lateral del núcleo ventral-posterior del tálamo. Transmite información propioceptiva consciente ${ }^{12,13}$.

- Tracto espinocerebeloso ventral: fibras ascendentes que, tras decusar en su origen, pasan por la porción anterior del cordón lateral en la médula espinal, pasan a través del bulbo raquídeo e ingresan a nivel del mesencéfalo al cerebelo a través del pedúnculo cerebeloso superior. Transmite información propioceptiva inconsciente, principalmente de los miembros inferiores ${ }^{12,13}$.
- Lemnisco espinal: es el conjunto de los tractos espinotalámico y espinotectal. Contiene información termoalgésica contralateral del tronco y las extremidades proveniente de la médula espinal y concluye en el núcleo ventral-posterior del tálamo ${ }^{12,13}$.

- Tracto piramidal: se trata de la vía corticoespinal cuando desciende a través de la pirámide en el bulbo raquídeo. Contiene información motora y está formado por las fibras descendentes de la neurona motora superior antes de su decusación ${ }^{12,13}$.

- Fascículo longitudinal medial: contiene fibras homolaterales y contralaterales provenientes de los núcleos vestibulares para terminar en los núcleos abducens, troclear y oculomotor. La información que transmite permite la coordinación de los movimientos oculares ${ }^{12,13}$. 
- Pedúnculo cerebeloso inferior: contiene aferencias cerebelosas provenientes de la médula espinal (a través del tracto espinocerebeloso dorsal), de los núcleos olivares y de los núcleos vestibulares ${ }^{12,13}$.

- Tracto espinal del trigémino: formado por fibras homolaterales de los nervios trigémino, facial, glosofaríngeo y vago que terminan en el núcleo espinal del trigémino, transmitiendo sensaciones generales de tacto, dolor y temperatura de la cabeza $^{12,13}$.

- Tracto solitario: formado por fibras de los ganglios inferior del hipogloso, vago y ganglio geniculado del nervio facial que terminan en el núcleo solitario. Transmite información del gusto y aferencias de reflejos relacionados con los nervios glosofaríngeo y vago ${ }^{12,13}$.

- Tracto trigeminotalámico ventral: contiene fibras ascendentes provenientes del núcleo espinal del trigémino que decusan para terminar en el tálamo contralateral. Transmite sensaciones generales de tacto, dolor y temperatura de la cabeza ${ }^{12,13}$.

- Fibras del nervio hipogloso: axones provenientes de las neuronas ubicadas en el núcleo del hipogloso; al salir por el surco preolivar forman el nervio hipogloso, encargado de la movilización de la lengua ${ }^{12,13}$

\section{Anatomía de la arteria cerebelosa posteroinferior}

La PICA es la rama más distal y prominente de la arteria vertebral, y tiene su origen en el segmento intracraneal de la arteria vertebral, en más del $40 \%$ de casos en el segmento medular lateral y casi en el $33 \%$ en el segmento premedular de la arteria vertebral (Fig. 3) ${ }^{12,14-16}$. En algunos casos la PICA se presenta como rama terminal de la arteria vertebral ${ }^{17}$. En un $5-20 \%$ de los casos la PICA tiene un origen extradura ${ }^{18}$.

Se encuentra presente en el $94-96 \%$ de los casos; se origina como arteria única en el $90-97 \%$ y duplicada en el 3-6\%15,19.

La PICA se divide, de acuerdo con lo propuesto por Lister, Rodríguez-Hernández y Rhoton, en cinco segmentos designados con la letra «p» minúscula y los números 1 a 5 . El segmento $p 1$, denominado segmento medular anterior, se encuentra en la porción anterior del bulbo raquídeo; el segmento p2, o segmento medular lateral, discurre en la parte lateral del bulbo raquídeo hasta el surco retroolivar, justo en el origen

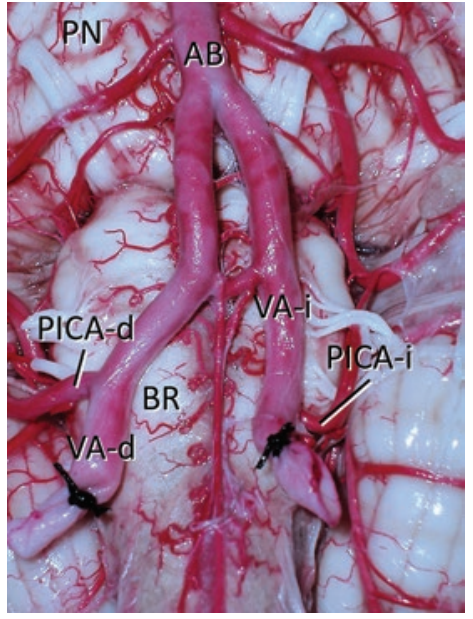

Figura 3. Origen de la arteria cerebelosa posteroinferior (PICA, posterior inferior cerebellar artery). $A B$ : arteria basilar; $B R$ : bulbo raquídeo; PICA-d: PICA derecha; PICA-i: PICA izquierda; VA-d: arteria vertebral derecha; $V A-i$ : arteria vertebral izquierda; $P N$ : puente.

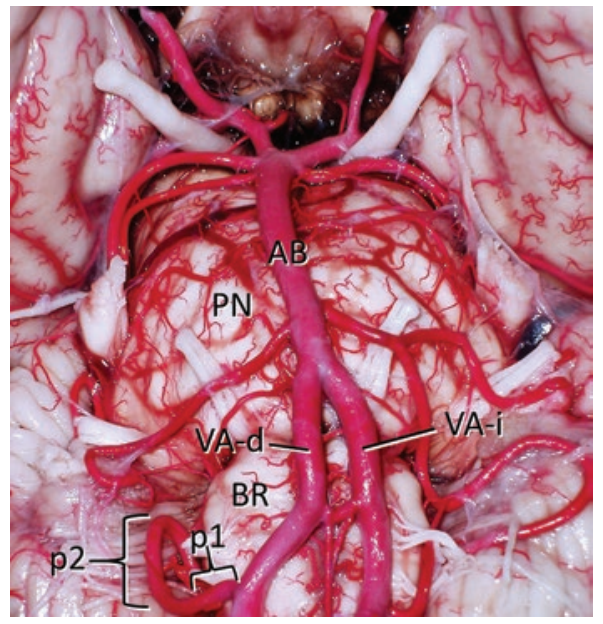

Figura 4. Segmentos iniciales de la arteria cerebelosa posteroinferior (PICA, posterior inferior cerebellar artery). Vista anterior del tallo cerebral. $A B$ : arteria basilar; $B R$ : bulbo raquídeo; $p 1$ : segmento medular anterior de la PICA; p2: segmento medular lateral de la PICA; $P N$ : puente; VA-d: arteria vertebral derecha; VA-i: arteria vertebral izquierda.

de los nervios glosofaríngeo, vago y accesorio (Fig. 4); el segmento p3, llamado segmento tonsilomedular, se relaciona con la mitad caudal de la amígdala cerebelosa; el segmento p4, o segmento telovelotonsilar, se encuentra en la hendidura entre la tela coroidea y el velo medular inferior rostralmente, y caudalmente el polo superior de la amígdala cerebelosa; finalmente, el segmento $\mathrm{p5}$, denominado segmento cortical, se encuentra en la superficie cortical de los hemisferios

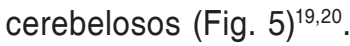

Sus territorios de irrigación incluyen la porción posterior de los hemisferios cerebelosos, la vermis inferior, los núcleos centrales del cerebelo y los plexos 

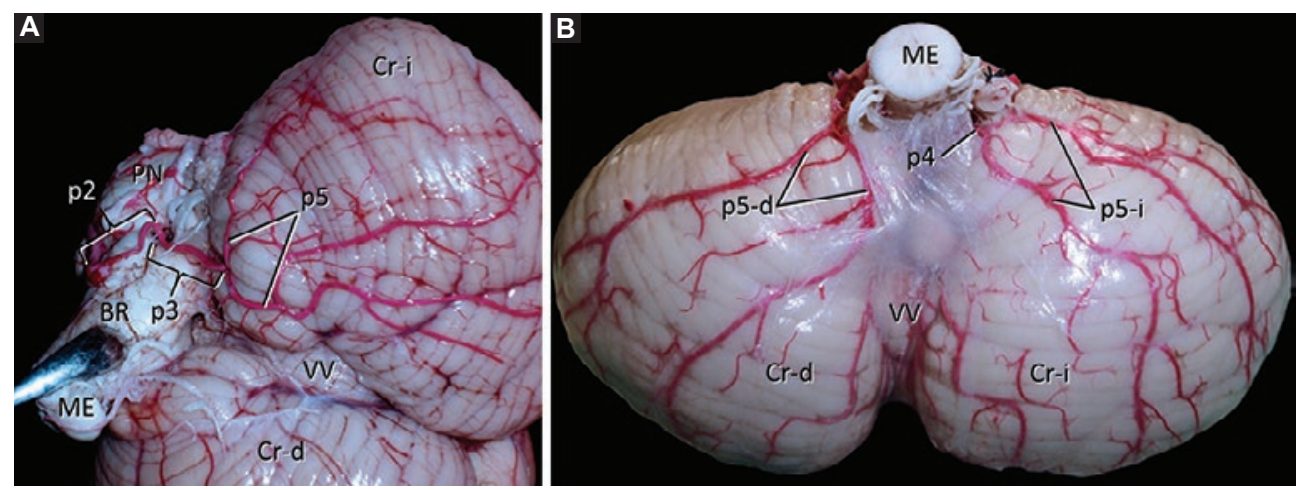

Figura 5. Curso de la arteria cerebelosa posteroinferior (PICA, posterior inferior cerebellar artery). A: vista inferolateral izquierda del cerebelo. B: vista inferior del cerebelo. BR: bulbo raquídeo; $\mathrm{Cr}$-d: hemisferio derecho del cerebelo; Cr-i: hemisferio izquierdo del cerebelo; $M E$ : médula espinal; p2: segmento medular lateral de la PICA; p3: segmento tonsilomedular de la PICA; p4: segmento telovelotonsilar de la PICA; 55 : segmento cortical de la PICA; p5-d: p5 derecho; p5-i: p5 izquierdo; PN: puente; VV: vermis ventral.
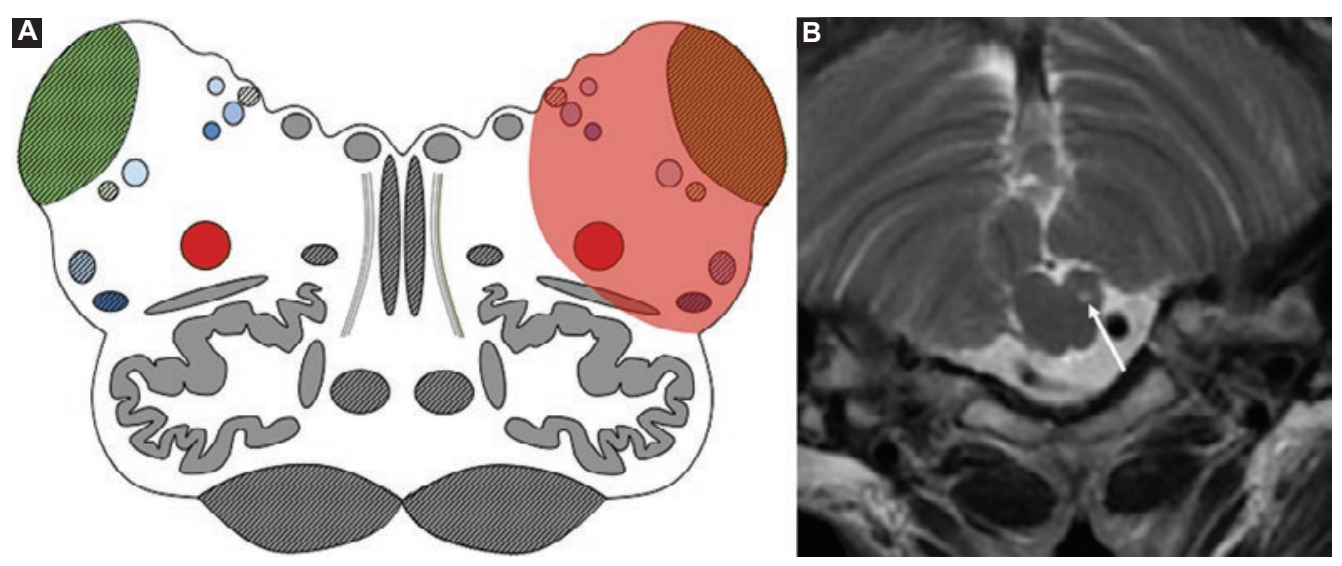

Figura 6. Territorio de isquemia en el síndrome de Wallenberg. A: esquema anatómico. La sombra en color rojo muestra el territorio de irrigación de la arteria cerebelosa posteroinferior izquierda. Los núcleos y la sustancia blanca afectada se muestran en color. B: imagen de resonancia magnética en T2 que muestra la isquemia (flecha blanca).

coroideos del cuarto ventrículo. Da origen a ramas medulares que irrigan la porción dorsolateral del bulbo raquídeo $0^{12,19,21}$.

\section{Manifestaciones clínicas}

Las manifestaciones clínicas clásicas del síndrome de Wallenberg se deben a la isquemia de las estructuras irrigadas por la PICA (Fig. 6) e implican a los núcleos vestibulares, el pedúnculo cerebeloso inferior, el núcleo espinal del trigémino, el tracto espinal del trigémino, el tracto espinotalámico, el núcleo ambiguo y las fibras simpáticas preganglionares descendentes ${ }^{12,13}$.

Con frecuencia (más del $60 \%$ de casos), en el síndrome de Wallenberg, además de la lesión directa del área medular lateral, hay infarto de otras áreas adicionales en el tallo cerebral ${ }^{22}$.

Estas manifestaciones incluyen vértigo, nistagmo, diplopía, síndrome de Horner, rubicundez y anhidrosis facial homolaterales, disfonía, disfagia, disartria, pérdida homolateral del reflejo nauseoso, ataxia homolateral, disgeusia homolateral, dolor y parestesia faciales homolaterales, pérdida o diminución homolateral del reflejo corneal, hipoalgesia y termoanestesia de tronco y extremidades contralaterales, hipoalgesia y termoanestesia facial homolateral $2,12,13,23-25$.

Además de los hallazgos clásicos, que pueden 0 no presentarse en su totalidad, el síndrome de Wallenberg se ha descrito como causa de epicrania fu$g_{a x^{26}}$, distonía cervicobraquial27, hipoventilación central $^{28}$, queratopatía neurotrófica y punteada ${ }^{4,23}$, manía ${ }^{29}$ y disfunción autonómica cardiovascular (con hipotensión ortostática) $)^{30}$.

\section{Correlación anatomoclínica de las manifestaciones neurológicas}

- Vértigo y nistagmo: se presentan tanto por lesión directa de los núcleos vestibulares (principalmente el inferior) que se encuentran en el 
territorio de irrigación de la PICA como por las vías vestibulares, en especial aquellas que comunican con la corteza vestibular parietoinsu$\operatorname{lar}^{2,12,13,23,31}$. El vértigo suele ser de características centrales en dirección a la mirada. Ambas manifestaciones clínicas pueden acompañarse de singulto, náuseas y vómitos secundarios ${ }^{2}$.

- Diplopía: por lesión pontina colateral y del fascículo longitudinal medial, estructuras involucradas en el movimiento ocular conjugado ${ }^{23}$.

- Síndrome de Horner, rubicundez y anhidrosis facial homolateral: la enoftalmia, la inyección conjuntival y la miosis características del llamado síndrome de Claude Bernard-Horner, así como la rubicundez y la anhidrosis facial, se producen por la lesión de las fibras simpáticas preganglionares descendentes que atraviesan el bulbo raquídeo 2,12,13,21,23.

- Disfonía, disfagia, disartria y pérdida homolateral del reflejo nauseoso: estas manifestaciones resultan de la lesión del núcleo ambiguo y de algunas fibras que dan origen a los nervios glosofaríngeo y vago. La disfagia puede ser grave en el $40 \%$ de los casos, y hasta el $100 \%$ de los pacientes presenta algún grado de disfagia; suele ser de corta duración y desaparecer casi en su totalidad en 4-10 semanas. De manera específica, la disfagia se presenta por la lesión de un generador central de patrones que controla centralmente el proceso de deglución 2,12,13,24,25.

- Ataxia homolateral: se presenta con tendencia a la lateralización homolateral y es consecuencia de la lesión cerebelosa directa por la oclusión de la PICA, por lesión de las fibras espinocerebelosas y por lesión de las fibras del pedúnculo cerebeloso inferior ${ }^{2,12,13}$.

- Disgeusia homolateral: ocurre por la lesión del tracto solitario y del núcleo solitario².

- Dolor y parestesia faciales homolaterales y pérdida o disminución homolateral del reflejo corneal: el tracto espinal del trigémino y el núcleo espinal del trigémino se ven afectados en el síndrome de Wallenberg, lo que explica el dolor y las parestesias homolaterales. Además, estas estructuras participan como vía aferente del reflejo corneal, necesario para el parpadeo durante el estímulo sensitivo corneal; dicha afectación explica la aparición de queratitis relacionada en algunos $\operatorname{casos}^{2,4,12,13,23 .}$.

- Hipoalgesia y termoanestesia de tronco y extremidades contralaterales: ocurre como resultado de la lesión del tracto espinotalámico que asciende a través del lemnisco espinal 2,12,13,23.

- Hipoalgesia y termoanestesia facial homolateral: por lesión del tracto espinal del trigémino y del núcleo espinal del trigémino $0^{2,12,13,23}$.

\section{Conclusión}

El síndrome de Wallenberg es la presentación clínica más frecuente de un evento vascular cerebral en la circulación posterior. Sus manifestaciones clínicas incluyen una gama diversa de signos y síntomas, la mayor parte de los cuales se explican fácilmente por la correlación anatómica con las estructuras afectadas. Su estudio es un reflejo claro de la importancia de la neuroanatomía en la aplicación clínica.

\section{Conflicto de intereses}

Los autores declaran no tener conflicto de intereses.

\section{Responsabilidades éticas}

Protección de personas y animales. Los autores declaran que los procedimientos seguidos se conformaron a las normas éticas del comité de experimentación humana responsable y de acuerdo con la Asociación Médica Mundial y la Declaración de Helsinki.

Confidencialidad de los datos. Los autores declaran que en este artículo no aparecen datos de pacientes.

Derecho a la privacidad y consentimiento informado. Los autores declaran que en este artículo no aparecen datos de pacientes.

\section{Bibliografía}

1. Zeidman LA, Mohan L. Adolf Wallenberg: giant in neurology and refugee from Nazi Europe. J Hist Neurosci. 2014;23:31-44.

2. Lui F, Bhimji SS. Wallenberg syndrome. StatPearls. Treasure Island (FL); 2017. Disponible en: https://www.ncbi.nlm.nih.gov/books/NBK470174/

3. Shenavandeh S, Petramfar P. Three atypical manifestations of granulomatosis with polyangiitis: lateral medullary syndrome, anterior cheek mass and melting scleritis of eye. Reumatologia. 2017;55:145-50.

4. Wu S, Li N, Xia F, Sidlauskas K, Lin X, Qian Y, et al. Neurotrophic keratopathy due to dorsolateral medullary infarction (Wallenberg syndrome): case report and literature review. BMC Neurol. 2014;14:231.

5. Canepa Raggio C, Dasgupta A. Three cases of spontaneous vertebral artery dissection (SVAD), resulting in two cases of Wallenberg syndrome and one case of Foville syndrome in young, healthy men. BMJ Case Rep. 2014 Apr 28;2014. pii: bcr2014203945.

6. Arora A, Kumar A, Raza MN. 'Legal high' associated Wallenberg syndrome. BMJ Case Rep. 2013 May 23;2013. pii: bcr2013009693.

7. Mullaguri N, Battineni A, Narayan A, Guddeti R. Cocaine induced bilateral posterior inferior cerebellar artery and hippocampal infarction. Cureus. 2018;10:e2576

8. Oks M, Li A, Makaryus M, Pomeranz HD, Sachdeva M, Pullman J, et al. Sarcoidosis presenting as Wallenberg syndrome and panuveitis. Respir Med Case Rep. 2018;24:16-8.

9. Stengl KL, Buchert R, Bauknecht H, Sobesky J. A hidden giant: Wallenberg syndrome and aortal wall thickening as an atypical presentation of a giant cell arteritis. BMJ Case Rep. 2013 Mar 1;2013. pii: bcr2012006994. 
10. Thomas VV, George T, Mishra AK, Mannam P, Ramya I. Lateral medullary syndrome after a scorpion sting. J Family Med Prim Care. 2017;6:155-7.

11. Malik MT, Kenton lii EJ, Vanino D, Dalal SS, Zand R. Lateral medullary ischemic infarct caused by posterior inferior cerebellar artery aneurysm. Case Rep Neurol. 2017:9:316-9.

12. Kiernan JA, Rajakumar N. Barr's The human nervous system: an anatomical viewpoint. $10^{\text {th }}$ ed. Philadelphia: Lippincott Williams \& Wilkins; 2014

13. Mtui E, Gruener G, Dockery P. Fitzgerald's Clinical neuroanatomy and neuroscience. $7^{\text {th }}$ ed. Philadelphia: Elsevier; 2016.

14. Rajasekhar SSSN, Aravindhan K, Tamgire DW. Duplicated and hypoplastic V4 segment of vertebral artery along with duplication of anterior inferior cerebellar artery: a case report. J Clin Diagn Res. 2017:11:AD03-5.

15. Pai BS, Varma RG, Kulkarni RN, Nirmala S, Manjunath LC, Rakshith S. Microsurgical anatomy of the posterior circulation. Neurol India. 2007;55:31-41.

16. Demartini ZJ de Oliveira TVH, Guimarães RMDR, Löhr A Jr, Koppe GL, Gatto LAM. Posterior inferior cerebellar artery origin over the C2 posterior arch. Pediatr Neurosurg. 2018;53:364-6.

17. Liu IW, Ho BL, Chen CF, Han K, Lin CJ, Sheng WY, et al. Vertebra artery terminating in posterior inferior cerebellar artery: a normal variation with clinical significance. PLoS One. 2017;12:e0175264.

18. Fine $A D$, Cardoso A, Rhoton AL Jr. Microsurgical anatomy of the extracranial-extradural origin of the posterior inferior cerebellar artery. J Neurosurg. 1999;91:645-52

19. Lister JR, Rhoton AL Jr, Matsushima T, Peace DA. Microsurgical anatomy of the posterior inferior cerebellar artery. Neurosurgery. 1982;10:170-99.

20. Rodríguez-Hernández A, Rhoton AL Jr, Lawton MT. Segmental anatomy of cerebellar arteries: a proposed nomenclature. Laboratory investigation. J Neurosurg. 2011;115:387-97.

21. Argente $\mathrm{H}$, Álvarez ME, editores. Semiología médica: fisiopatología semiotecnia y propedéutica: enseñanza-aprendizaje centrada en la persona. 2. ${ }^{a}$ ed. Buenos Aires: Médica Panamericana; 2013.
22. Kang HG, Kim BJ, Lee SH, Kang DW, Kwon SU, Kim JS. Lateral medullary infarction with or without extra-lateral medullary lesions: what is the difference? Cerebrovasc Dis. 2018;45:132-40.

23. Cidad P, Boto A, Del Hierro A, Capote M, Noval S, Garcia A, et al. Unilateral punctate keratitis secondary to Wallenberg syndrome. Korean J Ophthalmol. 2014;28:278-83.

24. Kim H, Lee HJ, Park JW. Clinical course and outcome in patients with severe dysphagia after lateral medullary syndrome. Ther Adv Neurol Disord. 2018;11:1756286418759864.

25. El Mekkaoui A, Irhoudane H, Ibrahimi A, El Yousfi M. Dysphagia caused by a lateral medullary infarction syndrome (Wallenberg's syndrome). Pan Afr Med J. 2012;12:92.

26. Jaimes A, García-Sáez R, Gutiérrez-Viedma A, Cuadrado ML. Case report: Wallenberg's syndrome, a possible cause of symptomatic epicrania fugax. Cephalalgia. 2018;38:1203-6.

27. Ogawa $T$, Shojima $Y$, Kuroki T, Eguchi H, Hattori N, Miwa H. Cervico-shoulder dystonia following lateral medullary infarction: a case report and review of the literature. J Med Case Rep. 2018;12:34.

28. Sivakumar K, Garcha M, Mehta D, Leary MC, Yacoub HA. Central hypoventilation: a rare complication of Wallenberg syndrome. Case Rep Neurol Med. 2018;2018:4894820.

29. Das P, Chopra A, Rai A, Kuppuswamy PS. Late-onset recurrent mania as a manifestation of Wallenberg syndrome: a case report and review of the literature. Bipolar Disord. 2015;17:677-82.

30. Huynh TR, Decker B, Fries TJ, Tunguturi A. Lateral medullary infarction with cardiovascular autonomic dysfunction: an unusual presentation with review of the literature. Clin Auton Res. 2018;28:569-76.

31. Yeo SS, Jang SH, Kwon JW. Lateral medullary syndrome following injury of the vestibular pathway to the core vestibular cortex: diffusion tensor imaging study. Neurosci Lett. 2018;665:147-51. 